\title{
Ultrahigh Ductile Gels Having Inter-Crosslinking Network (ICN) Structure*
}

\author{
Go Takada \\ Soft \&s Wet matter Engineering Laboratory (SWEL), \\ Department of Mechanical Systems Engineering, Graduate School of Science and Engineering, \\ Yamagata University, Jonan 4-3-16, Yonezawa, Yamagata 992-8510, Japan \\ Ruri Hidema ${ }^{\dagger}$ \\ Organization of Advanced Science and Technology, Kobe University, \\ Rokkodai 1-1, Nada, Kobe, Hyogo 657-8501, Japan, and \\ Soft 83 Wet matter Engineering Laboratory (SWEL), \\ Department of Mechanical Systems Engineering, Graduate School of Science and Engineering, \\ Yamagata University, Jonan 4-3-16, Yonezawa, Yamagata 992-8510, Japan \\ Hidemitsu Furukawa \\ Soft $\mathcal{G}$ Wet matter Engineering Laboratory (SWEL), Department of Mechanical Systems Engineering, \\ Graduate School of Science and Engineering, Yamagata University, \\ Jonan 4-3-16, Yonezawa, Yamagata 992-8510, Japan.
}

(Received 12 February 2012; Accepted 1 May 2012; Published 14 July 2012)

\begin{abstract}
Gels have unique properties such as low frictional properties, permeability and biocompatibility due to their high water content. In the last decade, several high-strength gels have been developed, which are promising for extending the application of gels as industrial materials. Previously we proposed novel ultrahigh ductile gels by defining inter-crosslinking network (ICN) structure. In this study, the mechanical properties of ICN gel was studied by experiments and theoretical estimations, and the mesh size of the internal network structure were determined. It is found that the Young's modulus of ICN gels calculated by experiments well matches with the theoretical estimations. The appropriate ICN gels achieves more than $61 \%$ increase in the ductility in comparison with normal poly $(N, N$-dimethylacrylamide) (PDMAAm) gels, by introducing the ICN structure. For the ICN gels, the density of chains calculated from the mesh-size is well consistent with the other densities calculated from the modulus and the water content, in comparison with the PDMAAm gels. [DOI: 10.1380/ejssnt.2012.346]
\end{abstract}

Keywords: Biophysics, medical physics, and biomedical engineering; Light scattering; Thermodynamic properties; Alkanes; Water; Hydro gels; Mechanical Properties; Network structure

\section{INTRODUCTION}

Gels have three-dimensional network structures of macromolecules including solvent, and by virtue of their high water content they have low friction properties, permeability, and biocompatibility. These are promising as a next generation of industrial materials, especially advantaged to medical applications. However, breaking stress in compression of usual gels is $0.1-0.4 \mathrm{MPa}$, which is quite low in comparison with the other hard materials, such as metal, ceramics, and plastics. The weak mechanical strength of gels had been a serious problem to use gels as industrial materials. This is why in recent years many trials were made to invent high-strength gels. In these trials, to develop epoch-making internal structures were important, such as Topological Gel, Nanocomposite Hydrogels, Double-Network Hydrogels, Tetra-PEG Gels, High-water-content mouldable hydrogels [1-8]. Especially, the Double-Network gels [3] achieves $30 \mathrm{MPa}$ of breaking stress in compression test at the maximum, which is the best record in the world. Since these rein-

*This paper was presented at the 6th International Symposium on Surface Science - Towards Nano, Bio and Green Innovation-, Tower Hall Funabori, Tokyo, Japan, December 11-15, 2011.

†Corresponding author: hidema@port.kobe-u.ac.jp forced gels could make a breakthrough for problems of weakness of gels, the gels attract much attention as new materials, and some trials have been conducted for an application as biomedical materials to artificial joint. These reinforced gels could achieve the high strength owing to their characteristic three-dimensional network structure of macromolecules. One of the most interesting points of gels research is how we control the mechanics of gels by the network structure.

In our previous research, Inter Cross-linking Network (ICN) structure was proposed as a first time to achieve novel gels having ultrahigh ductility $[9,10]$. Figure 1 shows the comparison of the internal structure of the ICN gel and other gels. In the case of Simple gels and Composite gels, the polymers have cross-linking points usually between the same types of polymers, and scarcely between the different types of polymers. The composite gel is sometimes categorized into an interpenetrated network. While, in the case of ICN gels, the intercross-linkers do not connect the same types of polymers, but connect the different types of polymers. Since the interaction between the same polymers is loose, the ICN network can deform largely in tensile test as shown in Fig. 1(d). This can be the reason for the properties of ultrahigh ductility $[10]$.

In this study, mechanical properties of ICN gels were precisely examined by experimentally and theoretically. Experimental Young's Modulus of the ICN gels $E_{\mathrm{t}}$ were 


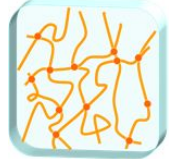

(a)

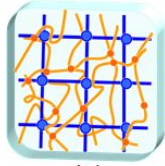

(b)
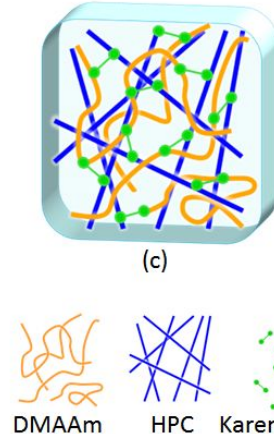

(c)
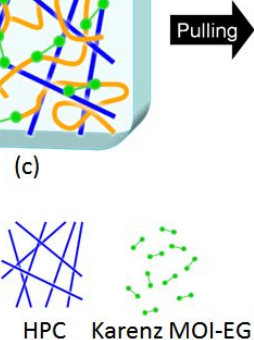

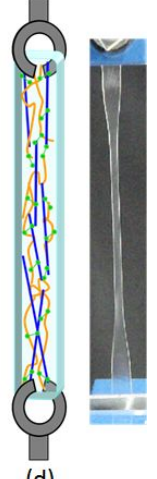

(d)
FIG. 1: Comparison of the internal structure of the ICN gel and the other gels. (a) shows a simple gel, which has single network structure. (b) shows a composite gel, which has crosslinking structure only between the same types of polymers. (c) shows the ICN gel which has crosslinking structure only between the different types of the polymer. (d) shows the schematic of the stretched inner structure of the ICN gel in tensile test.

determined by the stress-strain curve in tensile test and the theoretical Young's Modulus $E_{\mathrm{w}}$ were calculated by the equation of ideal rubber elasticity. By comparing both $E_{\mathrm{t}}$ and $E_{\mathrm{w}}$, internal structure of the ICN gels were considered. Further, a characteristic length of internal structure, i.e. the mesh size of the ICN gels was also determined by scanning microscopic light scattering (SMILS), which is a dynamic light scattering system specialized for analyzing the microscopic structure in gels [11]. Three types of the density of elastically effective chains, $\nu_{\mathrm{s}}, \nu_{\mathrm{w}}$, and $\nu_{\mathrm{t}}$ were calculated from the size of internal structure, the water content and the Young's modulus of tensile test. By comparing the three densities, the relation between the network structure and mechanical properties of the gels has been discussed.

\section{EXPERIMENTAL}

\section{A. Synthesis of the ICN gels}

Powder of the Hydroxypropyl cellulose (HPC, Wako Pure Chemical Industries ltd., Japan) and $\alpha$-keto (Wako Pure Chemical Industries ltd., Japan) was added to liquid $N, N$-dimethylacrylamide (DMAAm, TOKYO CHEMICAL INDUSTRY CO., LTD., Japan) and stirred till the HPC was solved. Here, HPC is monomer, $\alpha$-keto is photo initiator, DMAAm is monomer and also solvent. 2-(2Methacryloyloxyethyloxy)ethyl isocyanate (Karenz MOIEG, Showa Denko K. K.) as an intercross-linker was added and stirred for one hour to be reacted with the HPC. Then a little amount of water was added to stop the reaction of the Karenz MOI-EG and stirred for 30 minutes to be reacted with residual. The concentration of HPC was $0.5 \mathrm{~mol} \%$ to DMAAm, and that of $\alpha$-keto was $0.1 \mathrm{~mol} \%$. The molecular weight of HPC and the concentration of Karenz MOI-EG were controlled in order to vary the internal structure of ICN gels. The precise information is shown in Table I and our previous paper [10].
The substitutional ratio means how many Karenz MOIEG molecules exist for one repeating unit (pyranose ring) of HPC. When a Karenz MOI-EG exists for a pyranose ring, the substitutional ratio is $100 \%$. The pregel solution was poured into the mold that sandwiched the silicone spacer of $1 \mathrm{~mm}$ thickness between two pieces of glass plates, and was irradiated by UV lamp (the peak wavelength is $365 \mathrm{~nm}$ ) for about 9 hours, then the gels were prepared. After the polymerization, the gels were soaked in a large quantity of pure water more than 48 hours to be fully swollen. The viscosity of HPC is related to the molecular weight of the HPC.

\section{B. Tensile test of the ICN gels}

The tensile test was performed to measure stress-strain relation by the same method as our previous study [10]. The gels were punched out in the shape of dumbbell specimen (JIS K6251 Dumb-bell test pieces No. 8). The thickness of the sample was fixed at $3 \mathrm{~mm}$, the distance between the marked points was fixed at $16 \mathrm{~mm}$. The mechanical testing instrument STA-1150 (ORIENTEC) was used for the tensile test, and the test was performed at $100 \mathrm{~mm} / \mathrm{min}$ of crosshead speed. The tensile test was done in room temperature.

\section{Measurement by Scanning Microscopic Light Scattering (SMILS)}

The SMILS is the dynamic light scattering system specialized for gels or inhomogeneous solutions, as described in Ref. [11]. The ICN gels were observed by the SMILS with $532 \mathrm{~nm}$ laser beam. The distribution of the internal size, such as, mesh size of ICN gels can be calculated by the SMILS.

\section{RESULTS AND DISCUSSIONS}

\section{A. Mechanical properties of ICN gels}

The inset in Fig. 2 shows the stress-strain curves of the ICN gels in the tensile test. The numbers correspond to the sample numbers of Table I. It is found that the substitutional ratio clearly effects on the stress-strain curves. When the substitutional ratio is higher, the maximum breaking stress is higher, and the maximum strain is lower. When the substitutional ratio is lower, the maximum breaking stress is lower, and the maximum strain is higher. It implies that the relationship between the maximum stress and the maximum strain is trade-off. The precise value is shown in Table I. Sample (5) shows the largest maximum strain of 6.38 in all of the samples, which is $161 \%$ improved from the normal poly(DMAAm) (PDMAAm) gel. The water content of the ICN gels were well over $90 \%$, ranging from about 91.5 to $97.6 \%$.

Figure 2 shows the close up the stress-strain curves, whose region is indicated by the red rectangle in the inset of Fig. 2. The liner relationship between tensile stress $\sigma$ and strain $\varepsilon$ in this region are formulated with $\sigma=E_{\mathrm{t}} \varepsilon$. 
TABLE I: Experimental condition of ICN gels, maximum strain and water content.

\begin{tabular}{|c|c|c|c|c|c|}
\hline Sample Number & $\begin{array}{c}\text { Viscosity of } \mathrm{HPC}^{a} \\
(\mathrm{mP} \cdot \mathrm{s})\end{array}$ & $\begin{array}{c}\text { Ratio of } \text { MBAA }^{b} \\
(\mathrm{~mol} \%)\end{array}$ & $\begin{array}{c}\text { Substitutional ratio }^{c} \\
(\%)\end{array}$ & Maximum strain & $\begin{array}{l}\text { Water content } \\
\quad(\text { wt } \%)\end{array}$ \\
\hline PDMAAm Gel & No HPC & 0.05 & $\mathrm{~N} / \mathrm{A}$ & 3.58 & 94.5 \\
\hline ICN Gel (1) & $2.0-2.9$ & 0 & 100 & 1.86 & 91.5 \\
\hline ICN Gel (2) & $2.0-2.9$ & 0 & 30 & 3.24 & 94.7 \\
\hline ICN Gel (3) & $1000-5000$ & 0 & 100 & 1.84 & 91.9 \\
\hline ICN Gel (4) & $1000-5000$ & 0 & 30 & 5.11 & 96.6 \\
\hline ICN Gel (5) & $1000-5000$ & 0 & 10 & 6.38 & 97.6 \\
\hline
\end{tabular}

${ }^{a}$ Viscosity of HPC for $1 \mathrm{wt} \%$ aqueous solution at $25^{\circ} \mathrm{C}$. The higher viscosity corresponds to the higher molecular weight.

${ }^{b}$ Molar ratio of MBAA to DMAAm.

${ }^{c}$ The substitutional ratio means how many Karenz MOI-EG molecules exist for one repeating unit (pyranose ring) of the HPC. When a Karenz MOI-EG exists for a pyranose ring, the substitutional ratio is $100 \%$.

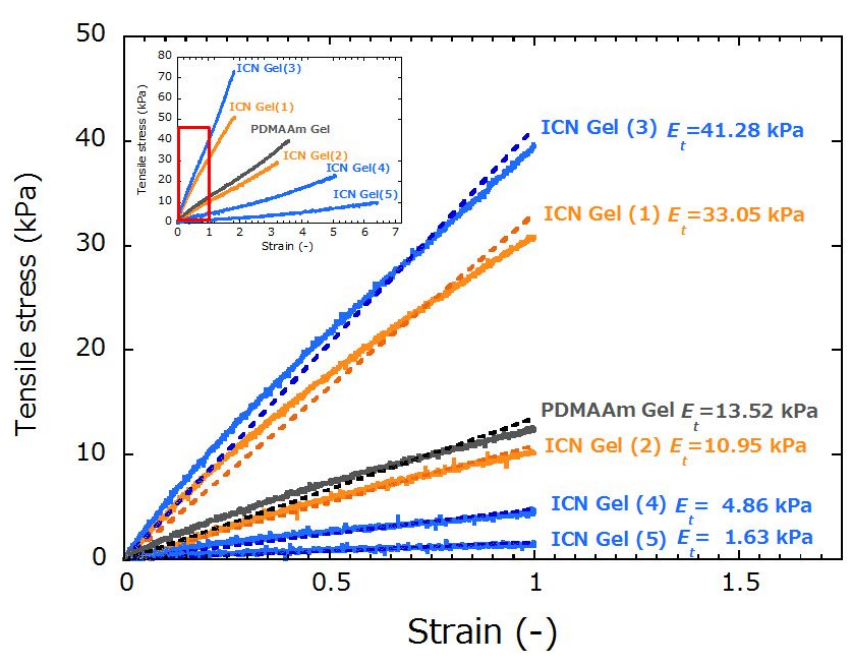

FIG. 2: Stress-Strain curve of the ICN gels. The inset is the whole curve, and the red rectangular is close up at the range of strain 0 to 1 , where the straight lines are fitted to the S-S curves to calculate the Young's modulus $E_{\mathrm{t}}$.

TABLE II: Comparison of Young's Modulus, $E_{\mathrm{t}}$ and $E_{\mathrm{w}}$, of each samples.

\begin{tabular}{ccccc}
\hline \hline $\begin{array}{c}\text { Code of the } \\
\text { sample }\end{array}$ & $\begin{array}{c}\text { Viscosity of } \\
\text { HPC }(\mathrm{mP} \cdot \mathrm{s})\end{array}$ & $\begin{array}{c}\text { Substitutional } \\
\text { ratio }(\%)\end{array}$ & $\begin{array}{c}E_{\mathrm{t}} \\
(\mathrm{kPa})\end{array}$ & $\begin{array}{c}E_{\mathrm{w}} \\
(\mathrm{kPa})\end{array}$ \\
\hline PDMAAm Gel & No HPC & N/A & 13.52 & 2.06 \\
ICN Gel (1) & $2.0-2.9$ & 100 & 33.05 & 31.36 \\
ICN Gel (2) & $2.0-2.9$ & 30 & 10.95 & 5.89 \\
ICN Gel (3) & $1000-5000$ & 100 & 41.28 & 30.3 \\
ICN Gel (4) & $1000-5000$ & 30 & 4.86 & 3.82 \\
ICN Gel (5) & $1000-5000$ & 10 & 1.63 & 0.89 \\
\hline \hline
\end{tabular}

$E_{\mathrm{t}}$ is the Young's modulus of the tensile test that was calculated from the slope of Fig. 2. Differently, the Young's modulus can also be estimated theoretically from the density of elastically effective chains $\nu_{\mathrm{w}}$, which is determined from the water content, based on the formula of entropic elasticity in the case of a perfect 4-functional network under the affine network assumption [12, 13], $E_{\mathrm{w}}=3 \nu_{\mathrm{w}} k_{\mathrm{B}} T$, where $k_{\mathrm{B}}$ is the Boltzmann constant and $T$ is the absolute

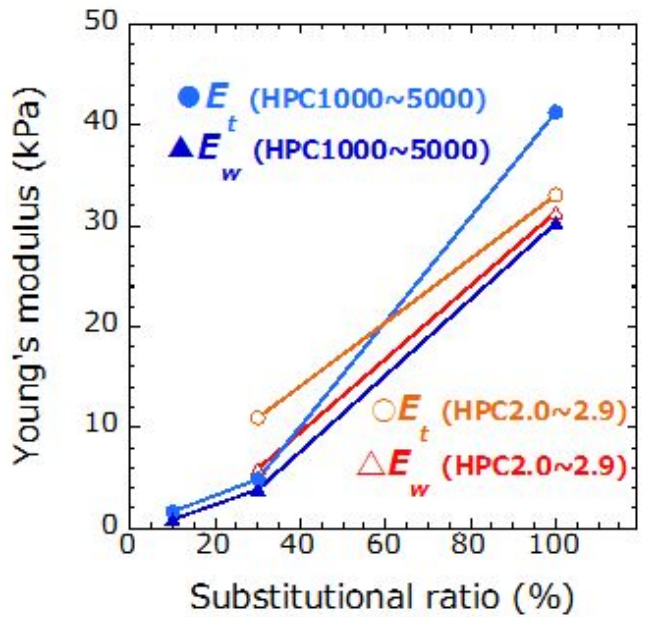

FIG. 3: The comparison between $E_{\mathrm{t}}$ and $E_{\mathrm{w}}$, depending on the substitutional ratio related to the crosslinking density.

temperature. The $\nu_{\mathrm{w}}$ is the density of elastically effective chains determined by the number of inter-crosslinking point divided by volume of gels after fully swelling. It is noted that $\nu_{\mathrm{w}}$ of fully (equilibrium) swollen gels is sometimes distinguished from $\nu_{\mathrm{w}}$ of as-prepared gels, if we consider both the states of preparation $\left(\phi_{0}\right)$ and measurement $(\phi)$. For example, $\nu=\nu^{\prime}\left(\phi_{0} / \phi\right)^{2 / 3}$, where $\nu$ and $\nu^{\prime}$ are the densities of the elastically effective chains in swollen and as-prepared states for $\theta$-solvent [13]. Here we treat only $\nu$ without due consideration for $\nu^{\prime}$, because all the measurement were done for fully swollen gels. Table II shows both the $E_{\mathrm{t}}$ and $E_{\mathrm{w}}$ of all the gels for comparison. It is noted that sample (5) indicated $1.63 \mathrm{kPa}$, which is the smallest $E_{\mathrm{t}}$. This $E_{\mathrm{t}}$ decreased by $12 \%$ in comparison with the normal PDMAAm gels.

Figure 3 shows the change in the $E_{\mathrm{t}}$ and $E_{\mathrm{w}}$ depending on the substitutional ratio. Fast of all, we see that $E_{\mathrm{t}}$ and $E_{\mathrm{w}}$ are well matched. It implies that the Young's modulus of the ICN gels is well controlled by the inter-crosslinking density. For the same viscosity of the HPC, it is found that the $E_{\mathrm{t}}$ and $E_{\mathrm{w}}$ increases as the substitutional ratio increases. Since the increase of the substitutional ratio means the increase of the inter-crosslinking point, this increasing behavior of the $E_{\mathrm{t}}$ is well understood by the 
TABLE III: Mesh size determined by the SMILS and the three kinds of inter-crosslinking densities, $\nu_{\mathrm{s}}, \nu_{\mathrm{w}}$, and $\nu_{\mathrm{t}}$, calculated from the mesh size of the SMILS, the water content, and the Young's modulus of the tensile test.

\begin{tabular}{ccccc}
\hline \hline $\begin{array}{c}\text { Code of the } \\
\text { sample }\end{array}$ & $\begin{array}{c}\text { Mesh size } \\
2 \xi(\mathrm{nm})\end{array}$ & $\begin{array}{c}\nu_{\mathrm{s}} \\
\left(1 / \mathrm{m}^{3}\right)\end{array}$ & $\begin{array}{c}\nu_{\mathrm{w}} \\
\left(1 / \mathrm{m}^{3}\right)\end{array}$ & $\begin{array}{c}\nu_{\mathrm{t}} \\
\left(1 / \mathrm{m}^{3}\right)\end{array}$ \\
\hline & & & & \\
PDMAAm Gel & 8.08 & $1.90 \times 10^{24}$ & $1.66 \times 10^{23}$ & $1.08 \times 10^{24}$ \\
ICN Gel (2) & 13.10 & $4.45 \times 10^{23}$ & $5.01 \times 10^{23}$ & $8.73 \times 10^{23}$ \\
ICN Gel (4) & 16.40 & $2.27 \times 10^{23}$ & $3.19 \times 10^{23}$ & $3.87 \times 10^{23}$ \\
\hline \hline
\end{tabular}

formula of the entropic elasticity theory, $E_{\mathrm{w}}=3 \nu_{\mathrm{w}} k_{\mathrm{B}} T$. Comparing $E_{\mathrm{t}}$ and $E_{\mathrm{w}}$ in detail, $E_{\mathrm{t}}$ is slightly larger than $E_{\mathrm{w}}$ for HPC1000 5000 in the high substitutional ratio. It implies that as the chain length of the HPC is larger, the entanglement among the polymer chains becomes so frequently that the entanglement possibly increases the apparent Young's modulus. By the way, we expect that the decrease of the inter-crosslinking point hopefully possibly causes the loose structure in the ICN gels as abovementioned. Namely, we expect that when the substitution ratio decreases, the stress concentration caused by the tensile test tends to be avoided due to its loose structure, which makes the gel deform largely. The experimental result well matches with our expectation.

\section{B. Size of internal structure}

The internal structure of the PDMAAm gel and the ICN gels (4) and (8) were measured by SMILS, and the characteristic mesh-size of network structure $2 \xi$ was determined as shown in Table III. Also, the density of elastically effective chains $\nu_{\mathrm{s}}$ is calculated from the $2 \xi$, as follows. If it is simply assumed that the structural unit in the ICN-gels is like a cube, the volume of the unit is calculated by $v=(2 \xi)^{3}$. If $\nu_{\mathrm{s}}$ corresponds to the density of the unit, $\nu_{\mathrm{s}}$ is calculated by $\nu_{\mathrm{s}}=(1 / v)$. Table III shows this calculated $\nu_{\mathrm{s}}$ and the $\nu_{\mathrm{w}}$ calculated from the water content in the Section III.1. Further, the other $\nu_{\mathrm{t}}$ is calculated from the Young's modulus of tensile test $E_{\mathrm{t}}$, by the formula of the entropic elasticity theory, $\nu_{\mathrm{t}}=E_{\mathrm{t}} /\left(3 k_{\mathrm{B}} T\right)$, as shown in Table III.

Figure 4 shows the dependence of $\nu_{\mathrm{w}}$ and $\nu_{\mathrm{t}}$ on $\nu_{\mathrm{s}}$. It is noted that the $\nu_{\mathrm{w}}$ and $\nu_{\mathrm{t}}$ are calculated from the mechanical properties of gels, while the $\nu_{\mathrm{s}}$ is calculated from the structure of gels. Thus Fig. 4 shows the relations between the structure and the mechanical properties. In Fig. 4 , the straight line of $\nu_{\mathrm{w}}=\nu_{\mathrm{s}}$ and $\nu_{\mathrm{t}}=\nu_{\mathrm{s}}$ implies the correspondences between the structure and the properties, due to the homogeneous network structure in gels, as shown in the inset illustration of network structure (b) in Fig. 4. In comparison with the PDMAAm gels, the plot of the ICN gels is close to the line. On one hand, when the network structure has the entanglement as shown in the inset (a) in Fig. 4, the entanglement prevents the swelling of gels and increases the modulus of gels, and thus the plot tends to be in the region of $\nu_{\mathrm{w}}>\nu_{\mathrm{s}}$ and $\nu_{\mathrm{t}}>\nu_{\mathrm{s}}$. On the other hand, when the network structure has many

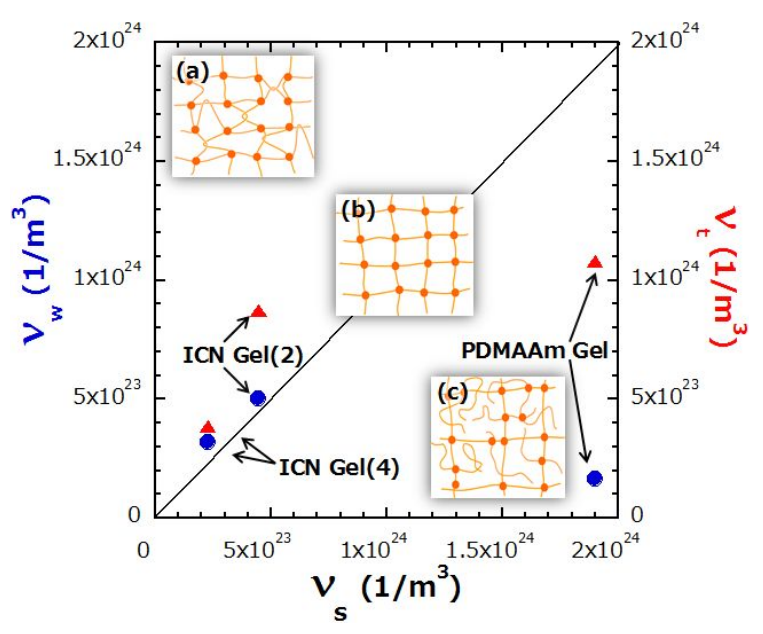

FIG. 4: The densities of effective chains, $\nu_{\mathrm{w}}$ (filled circle) and $\nu_{\mathrm{t}}$ (filled triangle), calculated from the water content and the Young's modulus $E_{\mathrm{t}}$, as functions of the other intercrosslinking density, $\nu_{\mathrm{s}}$, calculated from the mesh size $2 \xi$.

dangling chains with no entanglement as shown in the inset (c) in Fig. 4, the mesh size observed by the SMILS tends to be smaller due to the narrower distance between the chains. It causes the increase of the $\nu_{\mathrm{s}}$. Here the dangling chains do no work on the increase of the modulus and cause the increase of the swelling, and thus the plot tends to be in the region of $\nu_{\mathrm{t}}<\nu_{\mathrm{s}}$ and $\nu_{\mathrm{w}}<\nu_{\mathrm{s}}$. As the plot of the DMAAm gels is in this region, it implies that the DMAAm gels has many dangling chains inside.

\section{CONCLUSIONS}

We studied the mechanical properties and network structure of the ICN gels. The maximum strain of the ICN gels achieves 6.38 , i.e. $61 \%$ higher than normal PDMAAm gels. The relationship between the increase of maximum strain and maximum stress is trade-off, which should be overcome to develop tough and ductile ICN gels in future. The decrease of the inter-crosslinking density provides the effective stress relaxation and large deformation in the ICN gels. The Young's modulus calculated from tensile test $E_{\mathrm{t}}$ well matches with that calculated from the water content $E_{\mathrm{w}}$. The internal structure of ICN gels were observed by the SMILS. It implies that the ICN gels has more homogeneous and less dangling structure than the PDMAAm gels.

\section{Acknowledgments}

This study was supported in part by the Industrial Technology Research Grant Program in 2009 (Project ID: 09A25003a) from the New Energy and Industrial Technology Development Organization (NEDO) of Japan. This study was also supported in part by the Grant-in-Aid for Scientific Research (B) (Project No.: 22350097) from the Japan Society for the Promotion of Science (JSPS). 
[1] Y. Okumura and K. Ito, Adv. Mater. 13, 485 (2001).

[2] K. Haraguchi and T. Takeshita, Adv. Mater. 14, 1120 (2002).

[3] J. P. Gong, Y. Katsuyama, T. Kurokawa, and Y. Osada, Adv. Mater. 15, 1155 (2003).

[4] M. Malkoch, R. Vestberg, N. Gupta, L. Mespouille, P. Dubois, A. Mason, J. Hedrick, Q. Liao, C. Frank, K. Kingsbury, and C. Hawker, Chem. Commun. 26, 2774 (2006).

[5] T. Sakai, T. Matsunaga, Y. Yamamoto, C. Ito, R. Yoshida, S. Suzuki, N. Sasaki, M. Shibayama, and U. I. Chung, Macromolecules 41, 5379 (2008).

[6] Q. Wang, J. L. Mynar, M. Yoshida, E. Lee, M. Lee, K. Okuro, K. Kinbara, and T. Aida, Nature 463, 339 (2010).

[7] M. Guvendiren, and K. R. Shull, Soft Matter 3, 619
(2007).

[8] W. Yang, H. Furukawa, and J. P. Gong, Adv. Mater. 20, 4499 (2008).

[9] G. Takada, R. Hidema, and H. Furukawa, Proc. of the 46th Touhoku Branch Annual Meeting of the Jpn. Soc. Mech. Engineers 2011-1, 190 (2011).

[10] G. Takada, R. Hidema, and H. Furukawa, J. Solid Mechanics and Mater. Engin. 6, 169 (2012).

[11] H. Furukawa, K. Horie, R. Nozaki, and M. Okada, Phys. Rev. E 68, 031406 (2003).

[12] P. G. de Gennes, Scaling Concepts in Polymer Physics (Cornell University Press, Ithaca, New York, 1979).

[13] M. Rubinstein and R. Colby, Polymer Physics (Oxford University Press, New York, 2003). 